\title{
Desigualdades na prática esportiva e de atividade física nas macrorregiões do Brasil: PNAD, 2015
}

\section{Inequalities in sports and physical activity in the Brazilian macro-regions: PNAD, 2015}

\section{AUTORES \\ Vivian Hernandez Botelho ${ }^{1}$ iD \\ Andrea Wendt ${ }^{2}$ (ID \\ Eraldo dos Santos Pinheiro ${ }^{1,3}$ (D) \\ Inácio Crochemore-Silva ${ }^{1,2,3}$ (i) \\ 1 Universidade Federal de Pelotas, Escola Superior de Educação Física, Pelotas, Rio Grande do Sul, \\ Brasil. \\ 2 Universidade Federal de Pelotas, Programa de Pós-Graduaç̃̃o em Epidemiologia, Pelotas, Rio Grande do Sul, Brasil. \\ 3 Universidade Federal de Pelotas, Departamento de Desportos, Pelotas, Rio Grande do Sul, Brasil.}

\section{CONTATO}

Vivian Hernandez Botelho vivianbbotelho@gmail.com

Rua Luís de Camões, n. 625, Três Vendas. Pelotas, Rio Grande do Sul, Brasil. CEP: 96055-630.

DOI

10.12820/rbafs.26e0206

\section{(cc) BY}

Este trabalho está licenciado com uma Licença Creative Commons - Atribuição 4.0 Internacional.

\begin{abstract}
RESUMO
Estudos sobre desigualdades na prática de esporte e atividades físicas são uma importante ferramenta para embasar políticas públicas. Assim como abordagens de nível nacional, análises específicas de acordo com regiões se fazem necessárias. O objetivo deste estudo foi verificar as desigualdades em termos de sexo, cor da pele, área de residência e escolaridade na prática de esporte ou atividades físicas de acordo com as regiões do Brasil. Foram analisados os dados da investigação suplementar da Pesquisa Nacional por Amostra de Domicílios de 2015, uma amostra representativa da população de adultos brasileiros. As análises contemplaram a prática de esporte e atividades físicas durante o ano anterior à entrevista e análises simples e complexas de desigualdade foram realizadas de acordo com cada região do país. Homens, pessoas de cor de pele branca, moradores da zona urbana e com maior escolaridade apresentaram maior prática em todas as regiões. Em termos de magnitude das desigualdades, as regiões que apresentaram maiores desigualdades foram a norte (prática entre homens 12,9 pontos percentuais - p.p., maior que entre mulheres) e a sul (41,1 p.p. de diferença entre os grupos extremos de escolaridade e 13,4 p.p. entre zona urbana e rural). Pessoas de cor da pele branca apresentaram 4,9 p.p. a mais de prevalência em relação às pretas ou pardas, sem diferenças marcantes entre as regiões. Os resultados identificaram a presença de desigualdades sistematicamente na direção de grupos privilegiados social e economicamente em nossa sociedade em todas as regiões do país, embora magnitudes diferentes sejam observadas regionalmente.
\end{abstract}

Palavras-chave: Atividade motora; Saúde pública; Estudos transversais; Adulto.

\section{ABSTRACT}

Studies of inequalities in sports and physical activity are important tools for public policies. As important as national approaches, regional-specific analyses are required. The aim of this study is to assess inequalities in terms of gender, skin color, place of residence and levels of education according to the regions of Brazil. The National Survey of Households Sample (Pesquisa Nacional por Amostra de Domicilios, PNAD) carried out in 2015 with adults was analyzed. Sports and physical activity practice were assessed in the one-year period before the interview and simple and complex measures of inequalities were performed according to each region. Males, participants reporting white skin color, living in urban areas, and presenting higher education levels presented higher prevalence of sports and physical activity practice in all regions. In terms of inequalities magnitude, the regions presenting higher disparities were the north (practice among males 12.9 percentage points - p.p., higher than females) and the south (difference of 41.1 p.p. between education levels and 13.4 p.p. between urban and rural areas). Participants reporting White skin color presented 4.9 p.p. higher prevalence than their counterparts, without marked regional differences. The results identified systematic inequalities towards to the most social and economic privileged groups of our societies, and magnitudes of such inequalities were different according to the country regions.

Keywords: Motor activity; Public Health; Cross-sectional; Adults.

\section{Introdução}

A atividade física é uma prática importante na prevenção de doenças crônicas não transmissíveis, assim como para bem-estar físico, psicológico e social ${ }^{1}$. Além disso, um percentual importante das doenças não transmissíveis é atribuído a ausência de atividade física populacional ${ }^{2}$. Todavia, apenas $30,5 \%$ da população adulta brasileira relata praticar atividade física no $\operatorname{lazer}^{3}$ e a proporção de pessoas ativas pode variar de acordo com sexo, faixa-etária, condição socioeconômica, entre outros aspectos ${ }^{4}$.

Embora a prática esportiva seja um tipo de atividade física, muitas vezes ela é subestimada em coleta de dados, análises e abordagens que visam estudar 
ou promover atividade física em níveis populacionais. Ademais, considerando aspectos culturais, a prática esportiva pode ser um meio para implementação de intervenções designadas à promoção de comportamento ativo ao longo da vida. A escolha por realizar determinada atividade física ou esportiva muitas vezes está vinculada a motivação, variando de acordo com a idade e sexo entre os adultos ${ }^{5}$.

As práticas de atividade física e esporte estão fortemente relacionadas às condições de vida dos indivíduos. No Brasil a prática de atividade física e esporte por homens é superior quando comparado às mulheres e quanto maior o nível de escolaridade, maior é a prática de atividade física (43,6\% mais escolarizados e $22 \%$ menos escolarizados) e esporte $(49,4 \%$ mais escolarizados e $23 \%$ menos escolarizados) ${ }^{6}$. Do mesmo modo, maior é a prática de atividade física e esporte nos grupos com renda financeira familiar maior que três salários mínimos, aumentando ainda mais a prática para os que possuem renda entre cinco e 10 salários mínimos ${ }^{6}$.

Devido a questões históricas e suas dimensões continentais, o Brasil é um país com muitas desigualdades entre as regiões geográficas e a população em geral. Há estudos que buscam compreender como a população das referidas regiões do país se comportam em relação à prática de atividade física e esporte. Mielke et al. ${ }^{7} \mathrm{em}$ um estudo com dados da Pesquisa Nacional de Saúde (PNS) de 2013, analisaram as diferenças de prática de atividade física no lazer em adultos nas cinco regiões do Brasil e encontraram a maior prevalência de ativos no lazer na região Sudeste e entre os mais jovens e a região Centro-Oeste, apresentou os mais ativos no lazer as pessoas com maior escolaridade.

Um estudo realizado por Wendt et al. ${ }^{8}$, também com dados da PNS, verificou a distribuição dos tipos de prática de atividade física em adultos de acordo com as características sociodemográficas. A atividade mais relatada pelos participantes foi a caminhada, contudo diferenças entre as práticas conforme o sexo, nível de escolaridade, idade e região foram evidenciadas.

No Brasil, os sistemas de vigilância epidemiológica para fatores de risco disponíveis e que incluem a prática de atividade física são a $\mathrm{PNS}^{9}$, o sistema de Vigilância de Doenças Crônicas por Inquérito Telefônico (VIGITEL) $)^{4}$ e a Pesquisa Nacional por Amostras de Domicílios (PNAD) ${ }^{10}$. Além do monitoramento dos níveis de atividade física, essas estratégias são relevantes para que seja monitorado também as desigualdades sociais e econômicas nos níveis de prática. Nesse sentido, uma das lacunas ainda existentes na literatura é a identificação das desigualdades e suas magnitudes de acordo com as diferentes regiões do país e, em vista disso, o objetivo deste estudo foi verificar as desigualdades em termos de sexo, cor da pele, área de residência e escolaridade na prática de esporte ou atividades físicas de acordo com as regiões do Brasil.

\section{Métodos}

Este estudo apresenta um delineamento transversal com caráter descritivo. Foram analisados dados da investigação suplementar da PNAD de 2015, através dos indicadores referentes às práticas de esporte e atividade física. Esse inquérito de amostra representativa da população brasileira foi realizado pelo Instituto Brasileiro de Geografia e Estatística (IBGE) mediante parceria com o Ministério do Esporte ${ }^{10}$.

A amostra da PNAD foi desenvolvida de forma probabilística, na qual a seleção dos participantes originou-se a partir de três estágios, sendo eles: primário, referindo-se as cidades; secundário, aos setores censitários; e terciário, aos domicílios. Para coleta dos dados, em cada domicílio foi selecionado aleatoriamente um residente com mais de 15 anos de idade que respondeu um questionário com questões sociodemográficas e comportamentais. Conforme a Resolução No 466, de 12 de dezembro de 2012, do Conselho Nacional de Saúde, por a PNAD fazer parte do sistema de vigilância brasileiro, a utilização de seus dados não requer submissão a comitês de ética em pesquisa. Mais informações podem ser consultadas no relatório oficial ${ }^{10}$.

O questionário analisado no presente estudo teve como objetivo identificar quem praticou esporte ou atividade física de lazer no ano anterior a entrevista. É importante ressaltar que a diferença entre esporte e atividade física não foi especificada no questionário, ficando a critério do participante classificar de acordo com seu entendimento. Para este estudo foi utilizada como amostra adultos maiores de 18 anos e a análise das seguintes questões em que o sujeito deveria assinalar sim ou não: "O(A) Sr.(a) praticou algum esporte no seu tempo livre (fora do horário do trabalho e da educação física na escola)?"; "Além de todas as atividades de esporte a que se dedicou, praticou alguma outra que considerava somente como atividade física no seu tempo livre (fora do horário do trabalho e da educação física na escola)?”; "O(A) Sr.(a) praticou alguma atividade física, que não considera esporte, no seu tempo livre (fora do horário do trabalho ou da educação física 
na escola)?". Todas essas questões se referiam ao último ano e tinham como ponto de referência o dia da entrevista. No questionário da PNAD, a frequência e duração das atividades praticadas (atividade física e esporte) assumia nas opções de resposta categorias fechadas em períodos referentes a mês e semanas, o que demandaria uma séria de decisões arbitrárias para estimar a prática semanal de acordo com as recomendações atuais de atividade física. Assim, mantendo coerência até mesmo com o relatório oficial do inquérito, optamos por classificar os participantes da pesquisa apenas entre aqueles que relataram a prática de esportes ou atividades físicas no ano anterior.

Os dados foram analisados de forma descritiva e a partir da análise de desigualdades. As análises descritivas foram baseadas na prevalência de quem praticou esporte e atividade física no último ano, de acordo com sexo (masculino e feminino), cor da pele autorrelatada em branca, amarela, preta, parda ou indígena (devido ao extremamente baixo percentual de participantes auto declarados amarelos e indígenas, essas categorias foram excluídas das análises; pretos e pardos foram agrupados), área de residência (urbana e rural) e nível de instrução/escolaridade (sem instrução, fundamental incompleto, fundamental completo, médio completo e superior ou mais).

Para a análise de desigualdades foram utilizadas as diferenças para os estratificadores dicotômicos (sexo, cor de pele e área de residência) e o Índice Angular de Desigualdade (IAD) para o estratificador ordinal (escolaridade). O IAD é uma medida complexa de desigualdade que permite avaliação de estratificadores ordinais, como escolaridade, pois leva em conta todas as categorias da variável e não só os grupos extremos. $\mathrm{O}$ IAD varia de -100 a 100 e é expresso em pontos percentuais. Para esta medida o valor 0 (zero) representa a igualdade, valores positivos implicam em maior prevalência nos mais escolarizados e valores negativos, maior prevalência do desfecho nos menos escolarizados.

As diferenças são medidas simples de desigualdade calculadas através da subtração da prevalência em um grupo pelo outro e expressas em pontos percentuais (p.p.). No presente estudo a diferença de sexo foi calculada como a prevalência de atividade física ou esportiva nos homens menos a prevalência nas mulheres. Da mesma forma, a diferença de cor de pele foi calculada como a prevalência do desfecho nos participantes auto declarados brancos menos a prevalência nos autodeclarados pretos ou pardos. Para área de residência a diferença foi calculada como a prevalência do desfecho na zona urbana menos a prevalência na zona rural. Em termos de interpretação, o 0 (zero) significa a igualdade entre os grupos, valores positivos implicam em maior prevalência em homens, moradores de zona urbana e brancos e valores negativos implicam em maior prevalência em mulheres, moradores de zona rural e pretos/pardos. $\mathrm{O}$ erro padrão e subsequente intervalo de confiança dessa medida foi obtido através do método bootstrap. Além disso, as desigualdades foram analisadas para todo o país e de acordo com as diferentes macro regiões. Todas as análises foram realizadas no Stata 16.0 utilizando o comando svy em função do plano amostral.

\section{Resultados}

A amostra foi constituída por 67.649 adultos maiores de 18 anos, dos quais 52,5\% eram do sexo feminino, $46,2 \%$ se autorrelataram com cor de pele branca e $85,7 \%$ eram residentes da zona urbana. Referente a escolaridade, $32,8 \%$ possuíam ensino médio completo e a maior parte da amostra foi da região Sudeste com 43,9\% (Tabela 1).

A prevalência de pessoas que praticaram esporte ou atividades físicas no ano anterior à entrevista foi de $36,8 \%$. Avaliando todo o país, a prevalência de prática esportiva ou de outras atividades físicas foi de 40,6\% entre homens e 33,2\% entre as mulheres. Em termos de cor de pele, as pessoas que praticaram esporte ou atividades físicas foram 39,3\% entre aquelas que se auto identificaram brancas e $34,4 \%$ entre as que se auto identificaram como pretas ou pardas. Referente a área de residência, $38,1 \%$ das pessoas que eram moradores da zona urbana praticavam atividade física ou esporte enquanto a prevalência de prática na zona rural foi de $28,7 \%$. Em relação a escolaridade, a prática de esporte ou atividade física no ano anterior foi observada em $56,7 \%$ das pessoas que relataram ter pelo menos ensino superior completo praticaram. Esse percentual diminuiu para as demais categorias de escolaridade (44,4\% entre aqueles com ensino médio completo, $35,7 \%$ entre os participantes que tinha ensino fundamental completo, $26 \%$ entre quem tinha ensino fundamental incompleto e $20,5 \%$ entre os participantes sem instrução formal) - Tabela 1 .

A Figura 1 apresenta a medida de desigualdade absoluta, expressa em pontos percentuais sobre a diferença de praticantes de atividade física ou esporte (cálculo realizado referente a prática realizada por homens menos a prática realizada por mulheres) de acordo com o sexo e as cinco grandes regiões do Brasil. A região que 
Tabela 1 - Descrição da amostra e da prevalência de adultos maiores de 18 anos de idade que praticaram atividade física ou esporte. Brasil, PNAD 2015 ( $\mathrm{N}=67.649)$.

\begin{tabular}{|c|c|c|c|c|}
\hline & \multicolumn{2}{|c|}{ Descrição da amostra } & \multicolumn{2}{|c|}{$\begin{array}{l}\text { Prevalência de prática } \\
\text { esportiva ou atividade física }\end{array}$} \\
\hline & $\%$ & $\begin{array}{l}\text { Intervalo de } \\
\text { confiança }\end{array}$ & $\%$ & $\begin{array}{c}\text { Intervalo de } \\
\text { confiança }\end{array}$ \\
\hline \multicolumn{5}{|l|}{ Sexo } \\
\hline Masculino & 47,5 & $(47,1-48,0)$ & 40,6 & $(40,0-41,3)$ \\
\hline Feminino & 52,5 & $(52,0-52,9)$ & 33,2 & $(32,6-33,8)$ \\
\hline \multicolumn{5}{|l|}{ Cor da pele } \\
\hline Branca & 46,6 & $(46,2-47,1)$ & 39,3 & $(38,6-40,0)$ \\
\hline Preta ou parda & 53,4 & $(52,9-53,8)$ & 34,4 & $(33,9-35,0)$ \\
\hline \multicolumn{5}{|l|}{ Área de residência } \\
\hline Urbano & 85,7 & $(85,4-86,1)$ & 38,1 & $(37,6-38,6)$ \\
\hline Rural & 14,3 & $(13,9-14,6)$ & 28,7 & $(27,6-29,8)$ \\
\hline \multicolumn{5}{|l|}{ Escolaridade } \\
\hline Superior ou mais & 12,2 & $(12,0-12,6)$ & 56,7 & $(55,4-58,0)$ \\
\hline Médio completo & 32,8 & $(32,4-33,2)$ & 44,4 & $(43,5-45,2)$ \\
\hline $\begin{array}{l}\text { Fundamental } \\
\text { completo }\end{array}$ & 15,3 & $(14,9-15,6)$ & 35,7 & $(34,6-36,9)$ \\
\hline $\begin{array}{l}\text { Fundamental } \\
\text { incompleto }\end{array}$ & 30,6 & $(30,1-31,0)$ & 26,0 & $(25,3-26,7)$ \\
\hline Sem instrução & 9,1 & $(8,9-9,4)$ & 20,5 & $(19,3-21,7)$ \\
\hline \multicolumn{5}{|l|}{ Região } \\
\hline Norte & 7,9 & $(7,7-8,1)$ & 34,7 & $(33,6-35,9)$ \\
\hline Nordeste & 26,9 & $(26,5-27,3)$ & 34,9 & $(34,2-35,8)$ \\
\hline Sul & 15,0 & $(14,7-15,3)$ & 36,7 & $(35,9-37,4)$ \\
\hline Sudeste & 43,9 & $(43,5-44,4)$ & 39,7 & $(38,7-40,8)$ \\
\hline Centro-oeste & 6,3 & $(6,1-6,4)$ & 39,8 & $(38,4-41,3)$ \\
\hline
\end{tabular}

apresentou maior diferença de prática entre os sexos foi a Norte (12,9 p.p.), e as demais com pequenas variações entre as regiões (Sudeste 7,6 p.p., Sul 6,9 p.p., Nordeste, 6,3 p.p. e Centro-Oeste 5,9 p.p).

Referente a diferença da cor da pele na prática de atividade física ou esporte, no Brasil como um todo as pessoas com cor da pele branca relataram 4,9 p.p. a mais de prática no ano anterior comparado aos que se autodeclaram pretos ou pardos. Contudo, essa diferença analisada apresentou importante variação regional, sendo maior no Sul (5,8 p.p.) e menor no Norte $(1,1$ p.p.) - Figura 2.

$\mathrm{Na}$ Figura 3 é apresentada a desigualdade absoluta em termos de área de residência (urbana e rural) no relato de prática atividade física ou esporte de acordo com cada região do país. A prevalência em geral foi de

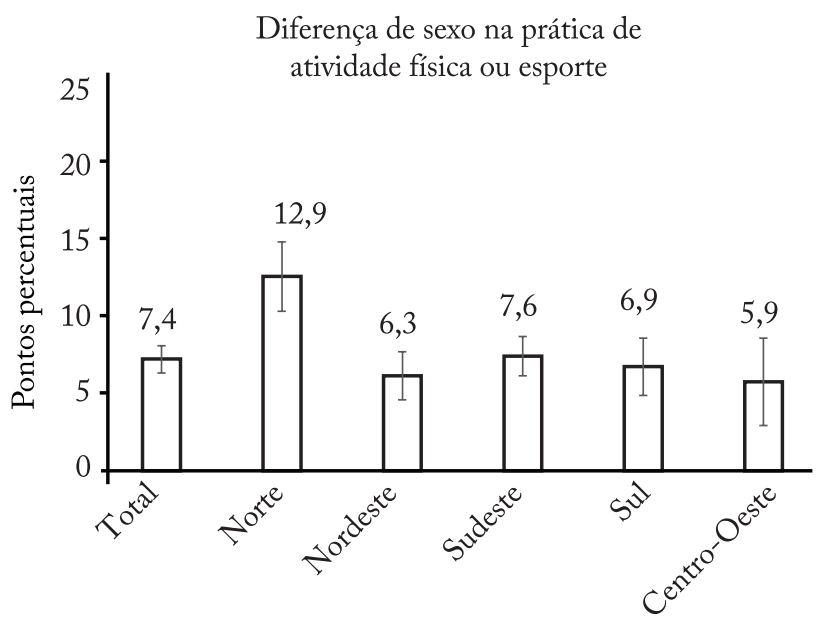

Figura 1 - Diferença de sexo* na prática de atividade física ou esporte expressa em pontos percentuais, conforme as regiões do Brasil. *Diferença de sexo = prática realizada por homem menos a prática realizada por mulher.

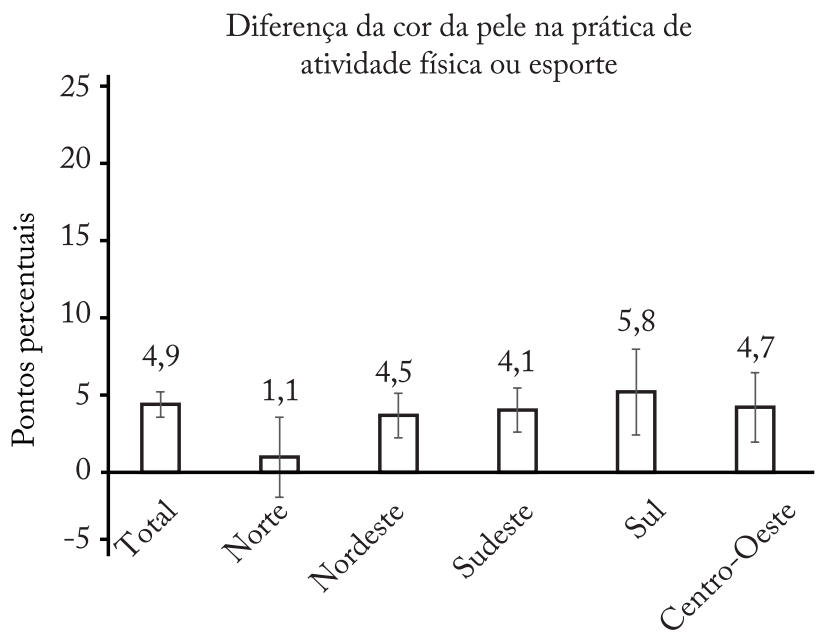

Figura 2 - Diferença da cor da pele* branca e preta ou parda na prática de atividade física ou esporte, conforme as regiões do Brasil. *Diferença da prática realizada por pessoas autorrelatadas de cor de pele branca menos a prática realizada por pessoas autorrelatadas de cor de pele preta ou parda.

6,7 p.p. maior em moradores da área urbana em comparação aos da zona rural. A região com maior diferença foi a Sul (13,4 p.p.), seguido pelo Centro-Oeste (12,9 p.p.), Sudeste (10,7 p.p.), Nordeste (7,5 p.p.) e Norte (7,2 p.p.).

Em relação as desigualdades entre os grupos de escolaridade, os grupos com maior escolaridade apresentaram 38,9 p.p. a mais de prática de esporte ou atividade física em comparação aos grupos com menor escolaridade. Os dados mostram que não houve grande variação observando a medida de desigualdade de acordo com as regiões. A maior desigualdade foi encontrada no Sul (41,1 p.p.) e a menor no Norte $(34,3$ p.p.), conforme observado na Figura 4. 


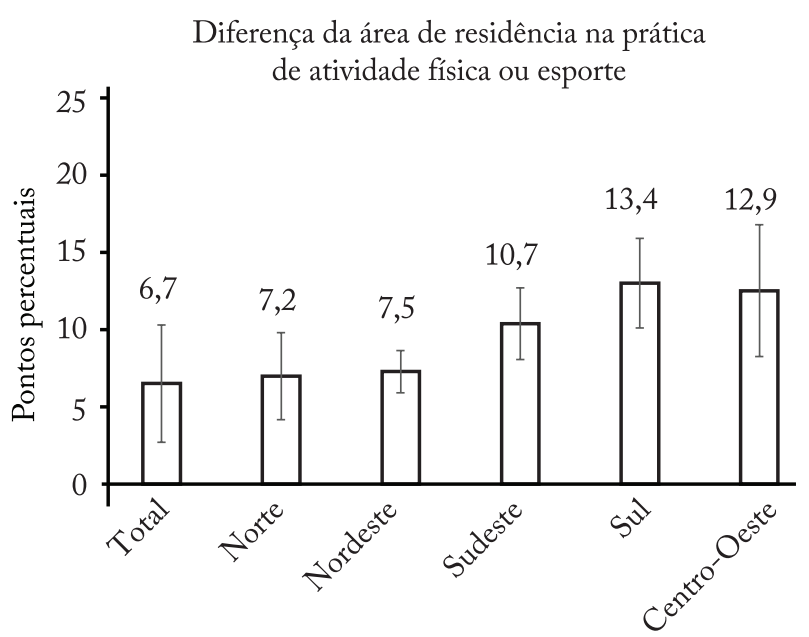

Figura 3 - Diferença de área de residência* dos praticantes de atividade física ou esporte, conforme as regiões do Brasil.

*Diferença dos moradores da área urbana em relação à área rural.

Desigualdade (IAD) na prática de atividade física ou esporte de acordo com escolaridade

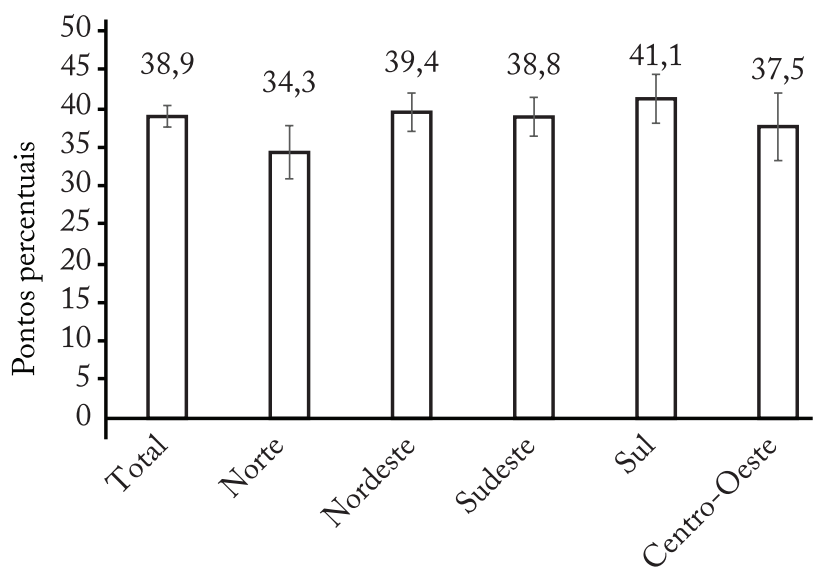

Figura 4 - Índice Angular de Desigualdade* (IAD) entre escolaridade e prática de atividade física ou esporte, conforme as regiões do Brasil.

* Diferença em relação aos grupos extremos de escolaridade no país.

\section{Discussão}

Os resultados deste estudo reforçam as evidências sobre as desigualdades na prática de atividade física ou esporte. A parcela da população com maior prevalência de prática é composta por homens, pessoas de cor de pele branca, residentes na área urbana, e com altos níveis de escolaridade em comparação a seus pares. Além disso, o presente estudo destaca que essas desigualdades foram observadas sistematicamente na mesma direção em todas as regiões do país. Quanto à magnitude, especificidades foram observadas e, em geral, maiores desigualdades entre os grupos comparados foram encontradas na região Sul e Norte.

Ao analisar as desigualdades na prática de atividade física ou esporte durante o período de lazer é necessária a reflexão permanente sobre o cenário ampliado das desigualdades sociais em nível nacional. Há uma grande disparidade em diversos fatores sociais entre as macrorregiões Norte/Nordeste e Sul/Sudeste/Centro-Oeste, a qual persiste em um longo período na história $^{11}$. Avaliando as desigualdades em saúde além da atividade física, destaca-se que geralmente são exatamente as pessoas do sexo masculino, brancas, residentes na zona urbana e com maior escolaridade que apresentam mais probabilidade de ter bons indicadores de saúde ${ }^{12}$. Os resultados observados no presente estudo podem ser considerados como consequência das desigualdades sociais no Brasil e ainda apresentam um papel potencializador da perpetuação dessas desigualdades.

Para praticar atividade física, grande parte das pessoas residentes em países de baixa renda deparam-se com várias barreiras ${ }^{13}$. Com a rápida urbanização dos países de baixa e média renda e suas demarcadas desigualdades sociais, os ambientes para a prática de atividade física não são igualmente distribuídos para a população e tampouco são planejados para oferecer possibilidades de prática de atividade física com segurança. Nesses países, a atividade física é majoritariamente praticada como meio de deslocamento, mas não por opção e sim por falta de escolha ${ }^{13}$. A atividade física de lazer, que é um direito básico do ser humano, é muitas vezes inacessível para essas populações.

As desigualdades sociais referentes a prática de atividade física de lazer já vêm sendo reportadas na literatura nacional e internacional. $\mathrm{O}$ estudo realizado por Chastin et al. ${ }^{14}$, examinou em níveis globais a prática de atividade física em adultos e evidenciou que em países de renda média e alta, as desigualdades econômicas internas estão associadas positivamente com maior prevalência de inatividade física. Além disso, os autores destacam que a desigualdade de gênero também tende a ser maior em países com maior desigualdade socioeconômica.

No contexto da América Latina, um estudo realizado com adultos em oito países latino-americanos, caracterizou os padrões sociodemográficos da atividade física e do tempo sentado ${ }^{15}$. Identificaram que a atividade física de lazer, deslocamento e tempo sentado variaram conforme o país, sexo e faixa-etária. Os níveis de atividade física de lazer e deslocamento foram maiores em homens do que nas mulheres e, os homens passam mais tempo sentados em relação a seus pares. Em ambas as atividades não foram encontradas diferenças significativas nos níveis socioeconômicos e de 
escolaridade, porém participantes com níveis socioeconômicos e escolaridade mais baixos, apresentaram maior percentual de inatividade física.

Por outro lado, estudos individualizados de países como Colômbia e Uruguai evidenciaram importantes desigualdades. No Uruguai, além das desigualdades de gênero, indivíduos pertencentes aos grupos de maior nível socioeconômico apresentaram prevalência de atividade física no lazer 20 pontos percentuais maiores que seus grupos de comparação ${ }^{16}$. Na Colômbia, dados da pesquisa nacional, realizada em 2010, identificaram que a prevalência de atividade física de lazer entre as mulheres de baixo nível econômico foi 7,4\%, enquanto entre as mais ricas foi $16,6 \%$, desigualdades também encontradas entre os homens $(22,2 \%$ entre os homens de maior nível socioeconômico e 31,8\% entre os mais ricos) ${ }^{17}$.

No Brasil, outros estudos como de Mielke et al. ${ }^{7}$ e Wendt et al. ${ }^{8}$, através dos dados da PNS verificaram que as práticas de atividade física de lazer são realizadas em maioria por homens, pessoas mais jovens e com maior escolaridade. Assim como Pontes et al. ${ }^{6}$, através dos dados na PNAD de 2015 evidenciaram que pessoas mais escolarizadas e com maior renda financeira familiar praticam mais atividade física e esporte. Diante disso, o presente estudo retrata as desigualdades referente a prática de atividade física e esporte com um olhar específico sobre as regiões do país, o que pode auxiliar na compreensão das mesmas.

A prevalência de atividade física de lazer no Brasil está aumentando paulatinamente ${ }^{18}$. No entanto, o aumento da atividade física na população geral não significa o aumento nos grupos que historicamente não tem as mesmas oportunidades, ou seja, não avançando nas reduções das desigualdades de prática de atividade física. Perante essas constatações, salientamos a importância do monitoramento das desigualdades sociais referente a atividade física e esporte ao longo do tempo ${ }^{19}$.

Estudos de base populacional, tradicionais na epidemiologia, e com ênfase sobre desigualdades, são importantes para orientar as políticas públicas que através de ações, devem promover a equidade. São informações à serviço do Estado, que por meio de interpretação e direcionamento, permite implementação de políticas que contemplem as necessidades de saúde da população ${ }^{20}$. A própria lei no 8080/1990, que estabelece o Sistema Único de Saúde (SUS), apresenta como essencial que as ações devem ser traçadas de acordo com as avaliações epidemiológicas da saúde da população ${ }^{21}$. São também oriundos do SUS, as estratégias de mo- nitoramento dos níveis de atividade física (VIGITEL, PNS e PNAD). Ademais, em termos de políticas principais que envolvem a promoção de atividade física no SUS, há o Núcleo Ampliado de Saúde da Família e Atenção Básica (NASF-AB)22, no qual o profissional de Educação Física é parte de equipes multiprofissionais que atuam conjuntamente com as equipes de saúde da família das unidades básicas de saúde em nível primário de atenção; e o Programa Academia da Saú$\mathrm{de}^{23}$, que abrange a criação de espaços públicos para a promoção de atividade física da comunidade local, ações sobre alimentação saudável, educação em saúde e práticas integrativas. Os dados da presente pesquisa contribuem para reforçar a necessidade dessas políticas que vem sendo ameaçadas nos últimos anos pela redução dos gastos públicos com saúde ${ }^{24} \mathrm{e}$ que, conforme as relatadas anteriormente, apresentam foco nas pessoas que mais precisam.

Nesse sentido, é importante destacar que grande parte dos estudos nacionais e internacionais contemplam as desigualdades de sexo e nível socioeconômi$\mathrm{co}^{7,8,14,15}$, contudo outras dimensões de desigualdades também precisam de maior investigação como cor de pele ${ }^{25} \mathrm{e}$ área de residência. Essas características têm um processo histórico de exclusão social e de esquecimento por parte das políticas públicas. Além disso, os estudos também necessitam observar as interseccionalidades, identificando grupos específicos a partir de múltiplas estratificações que apresentam piores indicadores de saúde e precisam ser foco de ações de inclusão/proteção social e promoção da saúde.

No presente estudo, analisamos as variáveis de atividade física e esporte, de acordo com a forma de coleta das informações e bancos de dados publicados. No entanto, não é recorrente observar a presença da prática esportiva nas ações que estimulam a prática de atividade física. No contexto brasileiro, a prática esportiva faz parte da cultura, sendo uma estratégia interessante para promover a saúde em virtude de uma abrangência de um maior número de pessoas. Além disso, o esporte tem um potencial que extrapola as intencionalidades de melhoria de indicadores de saúde para seus praticantes. Trata-se de uma manifestação da cultura corporal do movimento carregada de significados culturais e sociais que podem potencializar inclusive a maior adesão à atividade física em níveis populacionais ${ }^{10}$. A PNAD de 2015 remete a inclusão do esporte no estudo devido a importância do mesmo para toda a sociedade, assim como para os órgãos do governo, em que oferece con- 
tribuições para o planejamento, coordenação, supervisão e ações para democratizar a prática tendo em vista o fortalecimento do esporte nacional.

Este estudo apresentou algumas limitações, principalmente na forma como as variáveis de atividade física e esporte foram apresentadas no questionário. Os termos de atividade física e esporte não foram informados ao entrevistado, ficando a critério da interpretação do respondente. Se houvesse o fornecimento dessa informação, poderia haver uma exploração maior dos resultados e com direcionamentos específicos para o esporte e a atividade física. Outra limitação é referente ao extenso período recordatório, possibilitando relatos de práticas atípicas e hábitos de vida sem distinção. Em termos de aspectos positivos, consideramos que a coleta e análise da prática esportiva adicionada a atividade física, dado que o marcador do esporte pode ser importante para sinalizar o poder público estimular a população ser mais ativa através de outras formas. Outro destaque são as análises específicas das desigualdades com medidas simples e complexas e estratificada para cada região do país, com objetivo de identificar as especificidades existentes entre elas.

Os resultados do presente estudo identificaram a presença de desigualdades referente a prática de atividade física ou esporte sistematicamente na direção de grupos privilegiados social e economicamente em nossa sociedade (homens, pessoas de cor de pele branca, residentes na área urbana e, com altos níveis de escolaridade) em todas as regiões do país. Porém, maior amplitude das desigualdades em relação ao sexo foi observada na região Norte, e referente a cor da pele, área de residência e escolaridade na região Sul. Sendo assim, é necessário dar luz a essas desigualdades e fomentar que o poder público elabore programas de intervenções eficazes a promoção de atividades físicas de lazer e esporte para a redução do cenário observado, considerando as especificidades da população residente em cada região do país.

\section{Conflito de interesse}

Os autores declaram não haver conflito de interesse.

\section{Financiamento}

O presente trabalho foi realizado com apoio do Conselho $\mathrm{Na}$ cional de Desenvolvimento Científico e Tecnológico por meio de bolsa de iniciação científica.

\section{Contribuição dos autores}

Botelho VH, participou da concepção do manuscrito, interpretação dos dados e redação. Wendt $\mathrm{A}$, realizou a análise dos dados e revisão crítica do conteúdo. Pinheiro ES, participou da revisão crítica do conteúdo. Crochemore-Silva I, participou da concepção do manuscrito, análise, interpretação dos dados e revisão crítica do conteúdo.

\section{Referências}

1. Booth FW, Roberts CK, Laye MJ. Lack of exercise is a major cause of chronic diseases. Compr Physiol. 2012;2(2):1143-211.

2. Lee IM, Shiroma EJ, Lobelo F, Puska P, Blair SN, Katzmarzyk PT, et al. Effect of physical inactivity on major noncommunicable diseases worldwide: an analysis of burden of disease and life expectancy. Lancet. 2012;380(9838):219-29.

3. Lima MG, Malta DC, Monteiro CN, Souza NFS, Stopa SR, Medina LPB, et al. Correction: Leisure-time physical activity and sports in the Brazilian population: A social disparity analysis. Plos One. 2020;15(1):e0228095.

4. Brasil. Departamento de Análise em Saúde e Vigilância de Doenças Não Transmissíveis. Vigitel Brasil 2019: vigilância de fatores de risco e proteção para doenças crônicas por inquérito telefônico: estimativas sobre frequência e distribuição sociodemográfica de fatores de risco e proteção para doenças crônicas nas capitais dos 26 estados brasileiros e no Distrito Federal em 2019. Brasília: Ministério da Saúde; 2020. Disponível em: <http://bvsms.saude.gov.br/bvs/ publicacoes/vigitel_brasil_2019_vigilancia_fatores_risco. pdf> [2020 Outubro].

5. Molanorouzi, K, Khoo S, Morris T. Motives for adult participation in physical activity: type of activity, age, and gender. BMC Public Health. 2015;15(1):66.

6. Pontes SS, Silva AM, Santos LMS, Sousa BVN, Oliveira EF. Práticas de atividade física e esporte no Brasil. Rev Bras Promoç Saúde. 2019;32.

7. Mielke GI, Malta DC, Sá GBAR, Reis RS, Hallal PC. Diferenças regionais e fatores associados à prática de atividade física no lazer no Brasil: resultados da Pesquisa Nacional de Saúde-2013. Rev Bras Epidemiol. 2015;18:158-69.

8. Wendt A, Carvalho WRG, Silva ICM, Mielke GI. Preferências de atividade física em adultos brasileiros: resultados da Pesquisa Nacional de Saúde. Rev Bras Ativ Fís Saúde. 2019;24:e0079.

9. Pesquisa nacional de saúde: 2019: informações sobre domicílios, acesso e utilização dos serviços de saúde: Brasil, grandes regiões e unidades da federação / IBGE, Coordenação de Trabalho e Rendimento. Rio de Janeiro: IBGE; 2020. Disponível em:< https://biblioteca.ibge.gov.br/ visualizacao/livros/liv101748.pdf> [2021 Abril].

10. IBGE. Pesquisa Nacional por Amostra de Domicílios PNAD. Rio de Janeiro: IBGE; 2017. Disponível em: <https:// www.ibge.gov.br/estatisticas/sociais/populacao/9127pesquisa-nacional-por-amostra-de-domicilios.html?=\&t=oque-e> [2020 Junho].

11. Santos JAF. Classe social, território e desigualdade de saúde no Brasil. Saúde Soc. 2018;27:556-72.

12. Sousa JL, Alencar GP, Antunes JLF, Silva ZP. Marcadores de desigualdade na autoavaliação da saúde de adultos no Brasil, segundo o sexo. Cad Saúde Pública. 2020;36:e00230318.

13. Lambert EV, Kolbe-Alexander T, Adlakha D, Oyeyemi A, Anokye N, Goenka S, et al. Making the case for 'physical activity security': the 2020 WHO guidelines on physical activity and sedentary behaviour from a Global South perspective. BJSM. 2020;54:1447-8. 
14. Chastin SFM, Van Cauwenberg J, Maenhout L, Gardon G, Lambert EV, Van Dyck D. Inequality in physical activity, global trends by income inequality and gender in adults. Int J Behav Nutr Phys Act. 2020;17(142).

15. Ferrari LM, Kovalskys I, Fisberg M, Gómez G, Rigotti A, Sanabria YC, et al. Original research Socio-demographic patterning of self-reported physical activity and sitting time in Latin American countries: findings from ELANS. BMC Public Health. 2019;19(1723).

16. Brazo-Sayavera J, Mielke GI, Olivares PR, Jahnecka L, Crochemore-Silva, I. Descriptive epidemiology of Uruguayan adults'leisure time physical activity. Int J Environ Health Res. 2018;15(7).

17. Observatorio Nacional de Salud. Informe nacional de las desigualdades sociales en salud en Colombia [National report of the social inequalities in health in Colombia] (6th ed.). Instituto Nacional de Salud; 2015. Disponível em:< https://www.ins.gov.co/direcciones/ons/informes/6.\%20 desigualdades\%20sociales.pdf> [2021 Abril].

18. Vigitel Brasil 2018: Vigilância de fatores de risco e proteção para doenças crônicas por inquérito telefônico: estimativas sobre frequência e distribuição sociodemográfica de fatores de risco e proteção para doenças crônicas nas capitais dos 26 estados brasileiros e no Distrito Federal em 2018. Ministério da Saúde, Secretaria de Vigilância em Saúde, Departamento de Vigilância de Doenças e Agravos não Transmissíveis e Promoção da Saúde. - Brasília: Ministério da Saúde; 2019. Disponível em: < https://portalarquivos2.saude.gov.br/images/ pdf/2019/julho/25/vigitel-brasil-2018.pdf> [2020 Novembro].
19. Silva ICM, Knuth AG, Mielke GI, Lock MR. Promoção de atividade física e as políticas públicas no combate às desigualdades: reflexões a partir da Lei dos Cuidados Inversos e Hipótese da Equidade Inversa. Cad Saúde Pública. 2020;36:e0155119.

20. Henriques AS, Rosa LCS. As expressões das desigualdades de gênero na saúde. Braz J of Develop. 2020;6(3):11959-69.

21. Brasil. Lei no 8080, de 19 de setembro de 1990. Brasília: Presidência da República; 1990. Disponível em: <http://www. planalto.gov.br/ccivil_03/leis/18080.htm> [2020 Outubro].

22. Brasil. Ministério da Saúde. Secretaria de Atenção à Saúde. Departamento de Atenção Básica. Núcleo de Apoio à Saúde da Família: Vol. 1. Ferramentas para a gestão e para o trabalho cotidiano. Ministério da Saúde, Secretaria de Atenção à Saúde. Brasília: Ministério da Saúde; 2014.

23. Malta DC, Barbosa da Silva J. Policies to promote physical activity in Brazil. Lancet. 2012;380(9838),195-6.

24. Castro MC, Massuda A, Almeida G, Menezes-Filho NA, Andrade MV, Souza Noronha KVM, Rocha R, et al. Brazil's unified health system: The first 30 years and prospects for the future. Lancet. 2019;394(10195),345-56.

25. Araújo EM, Costa MCN, Noronha CV, Hogan VK, Vines AI, Áraújo TM. Desigualdades em saúde e raça/cor da pele: revisão da literatura do Brasil e dos Estados Unidos (19962005). Saúde Coletiva. 2010;7(40),116-21.

Recebido: $18 / 12 / 2020$

Aprovado: 05/06/2021

\section{Como citar este artigo:}

Botelho VH, Wendt A, Pinheiro ES, Crochemore-Silva I. Desigualdades na prática esportiva e de atividade física nas macrorregiōes do Brasil: PNAD, 2015. Rev Bras Ativ Fís Saúde. 2021;26:e0206. DOI: 10.12820/rbafs.26e0206 